\title{
¿Separados o juntos? \\ Contagio, escolaridad y tracoma en \\ Argentina (1884-1940)
}

\section{Separados ou juntos? \\ Contágio, escolaridade e tracoma na Argentina (1884-1940)}

\section{Apart or together? Infection, schooling and trachoma in Argentina (1884-1940)}

\author{
María Silvia Di Liscia ${ }^{1}$ \\ María Estela Fernández ${ }^{2}$
}

\begin{abstract}
RESUMEN
En el contexto de expansión de la educación pública en Argentina desde fines del siglo XIX, las iniciativas sanitarias provenientes de los poderes públicos incluyeron a la población infantil que concurría a los establecimientos educativos. Las preocupaciones por la salud de los niños en edad escolar derivaron entonces en el interés por distintas patologías, que, como en el caso del tratamiento del tracoma, implicaron estudios previos y discusiones en torno a las formas alternativas para enfrentar la enfermedad y a la disyuntiva de la segregación de sanos y enfermos. En esta línea, el presente artículo examina la conexión entre educación y tracoma entre 1884 y 1940 , a través del análisis de los aspectos generales de la atención sanitaria escolar y de la patología en el país desde su localización hasta los años veinte; de la experiencia particular de la instalación de una escuela especial para niños tracomatosos en el norte de Argentina; y de la proyección de un sistema
\end{abstract}

DOI: $10.1590 / 0104-4060.38204$

1 Universidad Nacional de la Pampa. Instituto de Estudios Socio-Históricos. Santa Rosa, La Pampa, Argentina. Cnel. Gil n. ${ }^{\circ} 353$ - 3er Piso. CP: L6300DUG.

2 Universidad Nacional de Tucumán. Faculdad de Ciencias Económicas. San Miguel de Tucumán, Argentina. Av. Independencia n. ${ }^{\circ}$ 1.900. CP: 4000. 
social y sanitario escolar basado en una legislación de carácter nacional a partir de la década de 1930.

Palabras clave: Argentina; tracoma; escolaridad; contagio.

\title{
RESUMO
}

No contexto da expansão do ensino público na Argentina, desde o final do século XIX, as iniciativas sanitárias por parte dos poderes públicos incluíram a atenção com a população infantil que frequentava as instituições de ensino. As preocupações com a saúde de crianças em idade escolar motivaram o interesse por várias doenças que, como no caso do tratamento do tracoma, foram baseadas em estudos já existentes e discussões sobre formas alternativas de lidar com a doença e o dilema da segregação de crianças saudáveis das doentes. Nesta linha, este artigo analisa a relação entre educação e tracoma entre 1884 e 1940, através da análise dos aspectos gerais da atenção com a saúde escolar e com esta patologia até os anos 1920; da experiência singular da instalação de uma escola para crianças tracomatosas no norte da Argentina, e da projeção de um sistema escolar social e de saúde com base na legislação de caráter nacional a partir de 1930.

Palavras-chave: Argentina; tracoma; escolaridade; infecção.

\begin{abstract}
In the context of public education expansion in Argentina since the end of the $19^{\text {th }}$ century the sanitary initiatives that came from the public powers took into account the child population that attended schools. The concern for children's health at school age ended up in the interest for different pathologies that, as in the case of the treatment for trachoma, implied previous studies and discussions around the alternative ways of dealing with the illness and the dilemma of segregation between healthy and ill kids. Thus, this paper examines the connection between education and trachoma from 1884 to 1940 through the analysis of general aspects of sanitary school attention and of the pathology until the 1920s; the particular experience of a special school that treated children who suffered from trachoma in de North of Argentina and the effect of a social and sanitary school system based on the national legislation from the 1930 s.
\end{abstract}

Keywords: Argentina; trachoma; schooling; infection. 


\section{Introducción}

Es de mañana, muy temprano. Apenas el sol asoma, ya los niños de Cuarto Grado de la Escuela Raúl Colombres, con sus blancos delantales, se preparan para saludar a la bandera en el patio. Los maestros los forman en apretadas filas y, a pesar del frío, cantan con entusiasmo el himno y también con algarabía entran a las pequeñas aulas donde repasan la geografía de Francia, más tarde resuelven problemas matemáticos y hacia el mediodía escriben laboriosamente una composición sobre el tema "Las estaciones del año". Muchos de los pequeños estudiantes están aprendiendo además el español, porque sus familias hablan árabe o italiano y han venido del otro lado del mar a trabajar en la zafra del azúcar o a cosechar el algodón, esforzándose día a día en ganar el pan. Nada distinto de otras aulas y otras escuelas argentinas de 1920, si no fuera porque, en los recreos, todos los alumnos deben asistir a una enfermería y se les colocan gotas en los ojos y, al salir, reciben una cartilla sanitaria con indicaciones sobre cómo lavarse las manos y la cara, desinfectar las vestimentas y evitar el contagio. Y porque, en este caso, todos los niños han sido diagnosticados como "tracomatosos".

En el cuadro que imaginamos, el estigma de la peligrosa enfermedad que infecta las conjuntivas y puede afectar la vista de manera definitiva llevando a los enfermos a la ceguera, llevó en Tucumán, una Provincia del Norte de Argentina, a establecer para los pacientes una institución educativa. El pretexto era de que los niños enfermos podían en contacto con otros sanos dispersar la enfermedad al resto de la familia, y de allí, infectar a toda la sociedad, haciendo peligrar la "sana raza" argentina. La excepcionalidad de esta medida permite reflexionar no sólo en relación a la particularidad de una enfermedad como es el tracoma, con su secuela de posibles ciegos y discapacitados, sino mucho más sobre la segregación de los enfermos, en este caso niños, así como sobre la integración laboral y social de los inmigrantes en el conjunto social argentino.

La conjuntivitis granulosa se produce cuando los ojos se infectan con la bacteria Chlamydia trachomatis y si no se cura apropiadamente, puede producir ceguera. Actualmente se la considera una enfermedad de la pobreza y aunque existe aún en regiones apartadas de Africa y Asia, donde escasea el agua y los recursos higiénicos son difíciles de obtener, fue erradicada a partir del uso masivo de terapias con sulfamidas y antibióticos en los años cuarenta. Se trató de un problema sanitario considerable en todo el mundo, justamente a la luz de la masiva movilidad de personas: el traslado de inmigrantes implicó su aparición en Argentina a finales del siglo XIX, aunque se diagnosticó por primera vez hacia 1903. 
Markel (2000) fue uno de los primeros en analizar tal situación en la inspección médica de inmigrantes judíos que intentaban ingresar a Estados Unidos con tracoma. En trabajos anteriores (DI LISCIA, 2012), hemos analizado la particular situación de los inmigrantes provenientes del Cercano Oriente o de Europa Central y Sur, a quienes se acusaba de introducir una patología de alto riesgo sin aportar al trabajo o a la configuración occidental argentina deseada y recientemente Gioa (2013) aportó en un estudio más profundo de las políticas inmigratorias respecto al tracoma en Argentina.

Asimismo, se avanzó sobre cómo los oftalmólogos hicieron suyo un campo profesional que les era esquivo o renuente a la especialidad, y se fortalecieron con la insistencia de que el sistema sanitario público generara un control más profundo de la enfermedad (DI LISCIA, 2013). También se analizó la composición y transformaciones de la atención de la salud en una de las más pujantes provincias argentinas del Norte, como lo era Tucumán a partir de la expansión de la industria azucarera, lo que atrajo ingentes modificaciones en la estructura demográfica y en la urbanización de pueblos y ciudades (FERNÁNDEZ, 2013). Y hay en la actualidad una profusa bibliografía sobre la incidencia de los higienistas primero y luego de los médicos sociales, en la construcción del sistema sanitario público argentino de los siglos XIX y XX (BELMARTINO, 2005; GONZÁLEZ LEANDRI, 2013).

Sin embargo, resta profundizar la conexión entre educación y tracoma, a la luz de la existencia en Tucumán de una Escuela de Tracomatosos, caso único que no se repitió en todo el país y es raro en el exterior. El debate de cómo proceder con los infectados en cuarteles, colegios y otras instituciones de participación masiva fue también una discusión sobre la segregación de enfermos y sanos, e incluía los derechos - muchas veces vulnerados - de los afectados quienes, no casualmente, también estaban apartados en virtud de su pertenencia social, "racial", étnica y religiosa. Pero a la vez, eran necesarios los brazos de más y más trabajadores, por lo que la reincorporación al mundo laboral se transformó en una labor imprescindible, en la que aunaban esfuerzos médicos, empresarios y miembros del conglomerado político.

En este artículo nos planteamos entonces un estudio de ese proceso histórico, que se inicia en 1884, cuando se ponen las bases del sistema educativo público argentino, durante el gobierno de Julio A. Roca, y se produce de manera paralela un incremento de la inmigración. Nuestro análisis finaliza hacia 1940, cuando se extiende la atención sanitaria y social a todos los escolares, a través de una legislación sancionada durante el gobierno conservador de Roberto M. Ortiz.

Se analizan sobre todo manuales y documentación estadística, así como la legislación producto de especialistas en oftalmología, agencias estatales sanitarias y educativas, a través del Monitor de Educación Común y de los Anales del 
Departamento Nacional de Higiene y de su Boletín, así como documentación específica en el caso particular de análisis. En primer lugar, se establecen los lineamientos generales de la atención sanitaria escolar y los aspectos generales de la patología en el país desde su localización hasta los años veinte; en segundo lugar, se estudia el establecimiento de una escuela para pequeños pacientes con tracoma en Tucumán y, finalmente, se analiza la particularidad de los años treinta y cuarenta, a raíz del establecimiento de un sistema social y sanitario más extendido.

\section{La salud visual de los escolares}

La sanción de la Ley n. ${ }^{\circ} 1.420$ “De Educación Común”, en 1884, extendió la obligatoriedad a un amplio conjunto de la población nacional. La educación pública penetró decididamente en la vida de los niños a partir de entonces y durante las siguientes tres décadas sin que el Estado tuviese competidores, por lo que tuvo éxito en imponer "[...] una cultura común, unificada, patriótica, democrática, con ingredientes cientificistas y espiritualistas" (ARMUS, 2014, p. 179). Desde finales del siglo XIX, los centros educativos argentinos, que eran en su mayoría públicos, estuvieron bajo la mirada higiénica, porque entre otras cuestiones se trataba de espacios compartidos por infantes durante largos períodos del año, donde hacían foco epidemias y se contagiaban las enfermedades crónicas. El máximo organismo a cargo de la educación, el Consejo Nacional de Educación, estaba por entonces subordinado a las decisiones de abogados y médicos (GONZÁLEZ LEANDRI, 2001) como Emilio Coni. Hacia 1887, este facultativo, presidente del Consejo, incluyó numerosas instrucciones sobre la limpieza, ventilación e iluminación de las aulas, las posturas y actividades de los pequeños y sobre el cúmulo de obligaciones de los maestros entre las que se contaban detectar piojos a diagnosticar paperas, rubeolas, completar las cartillas sanitarias e incluso vacunar (CONI, 1887).

Desde su fundación en 1882, el Monitor de Educación Común registra notas de médicos relacionadas con aspectos educativos y sobre la participación del Cuerpo Médico Escolar, un organismo creado tempranamente para velar por la salud de los escolares en la Capital Federal y en los Territorios Nacionales (ver como uno de los tantos ejemplos a Lema y Calderón, 1898). El interés por el tracoma apareció posteriormente, ya que las primeras preocupaciones por la vista de los escolares eran la detección temprana de miopías y otros problemas oftalmológicos que complicaban el aprendizaje (MARTÍNEZ; BUSTAMENTE, 1892).

En 1905, Reglamento del Cuerpo Médico Escolar preveía la necesidad de realizar un examen físico a los escolares que incluyera, entre otros aspec- 
tos, "una inspección ocular y auricular" (REGLAMENTO..., 1905). Y en ese mismo año, dos médicos del cuerpo realizaron una síntesis sobre la "higiene escolar" donde sistematizaban a todas las enfermedades y problemas que podían encontrarse entre los escolares, entre los cuales estaban clasificadas las de los ojos, como la "conjuntivis granulosa" y otras oftalmías purulentas e infecciosas (GUTIÉRREZ; DIEZ MORI, 1905).

Es preciso realizar aquí algunas precisiones respecto a la organización nacional de la salud y la educación. Hasta avanzado el siglo XX, el Departamento Nacional de Higiene tenía jurisdicción sobre los Territorios Nacionales y la Capital Federal, ya que en el resto del espacio nacional, las Provincias organizaron sus propios Consejos de Higiene. Las provincias, defendiendo su autonomía, se resistían al control más férreo del Departamento, que creía lógico trasvasar las fronteras en casos de epidemias o endemias recurrentes, pero tal situación fue siempre una fuente de conflictos entre el organismo nacional y los provinciales. En el caso de la educación, desde su creación en 1884, el Consejo Nacional de Educación ejercía sus funciones de igual manera que lo hacía el Departamento, tanto en Territorios Nacionales como la Capital.

En 1905, la Ley n. ${ }^{\circ}$ 4.874, denominada Ley Laínez, permitió al Consejo la creación de escuelas y colegios en las provincias, dando pie al ejercicio de políticas educativas de alcance nacional mucho más rápidamente que a políticas sanitarias de igual signo. El Departamento Nacional de Higiene realizaba periódicamente estadísticas en las provincias y si existían enfermedades que preocupaban a la seguridad pública, era obligación de los Consejos provinciales determinar su alcance y convocar al órgano nacional, pero es indudable que, como hemos analizado en otras oportunidades, la fortaleza de las instituciones educativas y su centralidad temprana, así como el número, alcance y significación de ambas profesiones, maestros y médicos, hacían muy diferente la aparición y el papel de ambas instituciones en el concierto nacional ${ }^{3}$.

Ahora bien, durante el Centenario de la Revolución de Mayo, las élites conservadoras gobernantes expusieron profusamente los avances argentinos, muchos ellos vinculados con la educación y con la higiene, como un sello del progreso. El tracoma, como otras patologías, había ingresado gracias al contacto con el exterior y era responsabilidad individual de inmigrantes, a quienes además se suponía poco adaptados a la comunidad nacional en virtud de su nacionalidad, "raza" y/o religión.

Dada la circulación de población a las áreas rurales y a que su dispersión se vincula a aspectos tanto ambientales (altas temperaturas y humedad) y sani-

3 Ver al respecto Di Liscia (2004) y un análisis comparativo más temprano en Rodríguez Leandri, síntesis general publicada en Billorou (2011). 
tarias (carencia de medidas higiénicas), la enfermedad comenzó a distinguirse en el Norte del país desde 1910.

El Departamento Nacional de Higiene, máximo órgano sanitario en el país por entonces, envió ese año al médico Enrique Demaría para estudiar el tracoma en las escuelas, quien también propuso un plan para evitarlo. Aparentemente no hubo mayor movimiento oficial durante varios años. Por entonces recién se iniciaba su reconocimiento y los funcionarios estaban inmersos en otros problemas sanitario-higiénicos, como las campañas antivariólicas y contra la malaria (DI LISCIA, 2012). La conexión con la inmigración haría pensar que la patología era más externa que interna, confiando su resolución a la extirpación de la "mala" migración.

Hacia 1921, el Cuerpo Médico Escolar contaba con una división de oftalmología en Buenos Aires, y se acreditan a partir de ese año y durante más de una década, el examen anual de 8.000 niños en las 300 escuelas porteñas (LIJÓ PAVÍA, 1922, 1937). Tal cuestión permitió intervenir tempranamente en la detección de la enfermedad y proporcionar los paliativos terapéuticos, evitando el avance del tracoma y sus serias consecuencias en la capital argentina, pero era mucho más difícil extirparla en el resto del país, donde había escasez de médicos y de centros especializados.

En 1924, se transforma el optimismo inicial de que la enfermedad sería eliminada simplemente con el control inmigratorio, toda vez que estaba ya extendida. Uno de los funcionarios del Departamento Nacional de Higiene realizó una encuesta dirigida a 2.000 médicos en todo el país, concluyendo que había numerosos casos en las provincias del Norte y en áreas pobladas con inmigrantes españoles, italianos, rusos y árabes ${ }^{4}$. A pesar de los esfuerzos en sistematizar las encuestas y en graficar la expansión del tracoma en un mapa general, no se realizó un intento por establecer de manera fehaciente la cantidad total de afectados. Para el médico encargado del Informe al Departamento, el tracoma debía ser de declaración obligatoria en todo el país y los enfermos serían obligados a tratarse. La infracción a tales hechos se castigaría con la pérdida de empleo y/o multas, cuestión que parece haber quedado en amenaza sin que se implementara en la realidad (BARBIERI, 1924, p. 24).

4 En en el Centro y Sur del país, la infección no parecía afectar a un número tan alto de personas: así, en el Norte de Santa Fé, una de las provincias con mayores índices de migración externa, de 1.000 enfermos examinados (Dispensario de Ceres), sólo 12 tenían tracoma y de 549 (Dispensario de Romang), 6 personas. En Córdoba, un estudio realizado desde 1912 a 1926 en el Hospital San Roque dio como resultado que, de 450 extranjeros, un 60\% tenía la enfermedad ocular y de 284 argentinos, un $38 \%$; en la división étnica, el autor exponía que las frecuencias más altas se daban entre los españoles (36\%), italianos (25\%) y árabes (24\%), concluyendo que el tracoma ha sido introducido por los extranjeros pero ha echado hondas raíces" (MAGGI ZABALIA, 1927, 1936). 
Para el organismo sanitario, la extensión de la enfermedad entre obreros portuarios de las grandes ciudades del Litoral y trabajadores rurales de los ingenios azucareros de Tucumán, por citar dos de los conjuntos más afectados, limitaría los brazos útiles y si el contagio llegaba a las familias de los trabajadores, fundamentalmente a niños en edad escolar, incidiría en la base misma de la nación. Los niños inmigrantes podían muy bien contagiar a los argentinos; por lo tanto, se requería una acción urgente.

A pesar de que se consideró al tracoma como una patología a estudiar y atender de manera más cercana, no hubo acciones del sistema público de manera inmediata que se replicaran en todo el país. El protagonista principal de las campañas fue un organismo privado, la Liga contra el Tracoma, organizada en Santiago del Estero en 1928 con el auspicio de la Cruz Roja Argentina y a partir de médicos locales con proyección nacional, como Enrique Demaría y los oftalmólogos Rodolfo Olle y Antenor Alvarez ${ }^{5}$.

La tarea de la Liga contra el Tracoma - especialmente, el diagnóstico - recibió el apoyo de enfermeras y visitadoras que realizaban las curaciones, estableciendo una metodología modélica para el tratamiento oftálmico del tracoma incluso fuera del país. De acuerdo a las investigaciones, el tracoma se hallaba difundido en todo el país y con cierta intensidad en la zona tropical del Norte, en las provincias de Santiago del Estero, Jujuy, Salta y Tucumán a raíz de la inmigración "siria y europea". En un examen realizado a 6.752 niños en Santiago del Estero, un 14,7\% tenían la enfermedad (DI LISCIA, 2010).

Sintetizando, en Buenos Aires era la inspección de la división de oftalmología del Cuerpo Médico Escolar la que vigilaba a los niños en las mismas aulas. En Santiago del Estero, esa tarea la realizaba sobre todo la Liga contra el Tracoma, que además la extendía a los trabajadores rurales. En otros puntos del Norte del país, la situación mereció una actuación diferente de los sectores gobernantes coaligados con los profesionales médicos, para resolver una situación que se visualizaba de manera alarmante y que de extenderse, podía afectar sus intereses económicos y hacer peligrar el progreso provincial.

\section{¿Jardín de la República o paraíso del tracoma?}

Tucumán, que por sus maravillosos paisajes y condiciones naturales de clima y tierra fértil se denomina "El Jardín de la República", no es una provincia

5 Sobre la importancia de la intervención de médicos oftalmólogos ver Argañaraz (1932) y Demaría (1938) en Di Liscia (2013). 
más del Norte argentino. En su capital, San Miguel de Tucumán, se declaró en 1816 la independencia y desde finales del siglo XIX, en el marco de un proceso político basado en el predominio de una élite que participaba del sistema de poder nacional, fue escenario de profundas transformaciones económicas, demográficas, sociales y culturales vinculadas en gran medida a la especialización agroindustrial azucarera, que requería ingentes cantidades de mano de obra sana y, a la vez, proletarizada y subordinada a las decisiones de los empresarios (BRAVO; CAMPI, 2000; CAMPI, 1999).

En este contexto, con el fin de garantizar la procreación y favorecer las condiciones de salud de futuras generaciones, la salud del niño en edad escolar fue uno de los aspectos incorporados a las políticas sanitarias, donde el discurso de las autoridades confluía en la necesidad de difusión de preceptos higiénicos, la vacunación preventiva, los controles en las escuelas, el rol de la alimentación y la mejora en las condiciones materiales.

La niñez como etapa crucial de la vida, vulnerable a las enfermedades y con menores posibilidades de sobrevivencia - sobre todo en los sectores más desprotegidos -, constituía uno de los tópicos que integraban la agenda de problemas sociales desde la doble vertiente privada y estatal. Asimismo, era el momento para intervenir y formar nuevos ciudadanos, que fueran también mano de obra apta para el trabajo productivo. De este modo, se intentó fortalecer el cuidado de la salud de los niños desde distintos espacios, incluidos los establecimientos educativos de la Provincia ${ }^{6}$.

En el marco de un proyecto nacional de mayores dimensiones que tendía a la expansión de la educación pública, en Tucumán se instalaron nuevas escuelas y se renovaron planes de estudio ${ }^{7}$. Las preocupaciones por la salud escolar se extendieron a reglamentos que estipulaban como condiciones de ingreso a las escuelas que los niños debían estar vacunados, no padecer de enfermedad contagiosa y probar su estado de salud con una certificación médica (REGISTRO..., 1901, p. 496-498; p. 508-509). La ley de educación común de 1914 incluía nociones sobre el "desarrollo moral, intelectual y físico del niño", la inspección médica en las escuelas y la obligación de enseñar a “[...] conservar

6 Otros espacios fueron el Hospital de niños y la Casa Cuna y Asilo Maternal, fundados en 1899 y 1905 por dos instituciones benéficas, la Sociedad de Beneficencia y la Conferencia de San Vicente de Paul, que se sostenían a través de contribuciones privadas y subsidios estatales.

7 También se creó un jardín de infantes que buscaba favorecer el crecimiento de los niños con prácticas gimnásticas en la etapa de dos a cinco años (LÁZARO, 1968). En relación al rol de los docentes y a la idea de alejar los peligros de contagio entre los alumnos se difundió un texto titulado "Higiene escolar", valorado por su importancia científica y por su utilidad como primer texto de higiene para las escuelas de la provincia (LIBRO..., 1897, p. 25-30). 
y robustecer la salud corporal y mental; a recuperar la salud en los casos de enfermedad leve; a prestar los primeros auxilios en los casos de accidente"

Poco tiempo después, estos principios se corporizaron en proyectos como la Ley de enseñanza obligatoria de higiene infantil y la creación del Cuerpo Médico Escolar - integrado por dos facultativos en la Capital y por el médico de policía en los departamentos del interior - con el objetivo de asesorar en asuntos de higiene y salud a niños y maestros. Sus funciones comprendían la inspección de locales; la realización de exámenes médicos y conferencias sobre temas de higiene, primeros auxilios y síntomas de enfermedades infectocontagiosas; y la asistencia gratuita a docentes y alumnos de probada pobreza ${ }^{9}$.

Como se ha señalado para el ámbito nacional, la problemática de la salud pública refleja transformaciones y nuevas orientaciones a partir de la década del 1920, que inaugura una etapa de mayor intervención del Estado en la provisión de servicios (BELMARTINO, 2005, p. 59). En Tucumán, los nuevos conceptos vinculados a la asistencia social y a la consideración de nuevos problemas y enfermedades, podrían asociarse a algunos cambios en relación a la salud de los niños en edad escolar, que concitaron a las instituciones sanitarias y educativas. Este fue el caso de las intervenciones para enfrentar el tracoma, donde la mirada puesta en los ojos de los niños implicó, de parte de las autoridades médicas, detectar la dimensión del problema, identificar el peligro de contagio y programar acciones con las autoridades del área de educación, que alcanzaron su máxima expresión en la creación de una Escuela para tracomatosos en 1920.

Tal vez el antecedente más directo de esta iniciativa derivó de una epidemia de conjuntivitis catarral purulenta en 1915, que por su carácter crónico y los efectos que producía en la visión podía, de acuerdo a los médicos de entonces, desencadenar en una conjuntivitis granulosa o "tracoma", que atacaba especialmente a los niños. En este contexto se realizó el primer estudio sobre la

8 Los planes de estudio incluían contenidos de higiene: de $1 .^{\circ}$ a $3 .^{\circ}$ grado, aseo personal, limpieza, alimentos, agua; de $4 .^{\circ}$ a $6 .^{\circ}$ grado, higiene de la digestión, órganos, profilaxia de enfermedades infectocontagiosas, higiene infantil (VALDEZ DEL PINO, 1920, p. 157-159; p. 165). Un tema también vinculado con la salud escolar fue el de la vacunación obligatoria, en tanto según la ley aprobada en 1911, los controles se harían con el Consejo de Educación, las normativas se incluirían en los programas de estudio, y no se aceptarían en los establecimientos alumnos que no estuvieran vacunados (GARCÍA, 1934, p. 939-946).

9 La ley del año 1914 también incluía un registro de “anormales físicos y orgánicos”, sobre los que se llevaría un control de tratamiento y resultados, con la posibilidad futura de una escuela especial para esos niños (GARCÍA, 1934, p. 681-683). Esta inclusión corrobora la tendencia a catalogar y, como analiza Rodríguez de Anca, la diferenciación de las categorías de "debilidad" y "anormalidad" mostraban la preocupación por los "retrasados pedagógicos", el peso dado a los factores hereditarios y la necesidad de intervención educativa (RODRIGUEZ DE ANCA, 2004, p. 31). 
difusión del tracoma en el interior de la provincia, del que resultaron informes detallados y propuestas para enfrentar la patología.

Las inspecciones, a cargo del médico especialista A. Calamatta, se hicieron en 19 escuelas y concluyeron que la enfermedad era endémica y llegaba a proporciones alarmantes - el mayor porcentaje alcanzado en uno de los establecimientos era de $43,9 \%$ y el menor de $24,07 \%$. De esta realidad surgía la necesidad de realizar desinfecciones, instar a la obligación de cura por parte de los padres, a la admisión escolar con certificado médico y al "pronto alejamiento de las escuelas de todos los niños enfermos" ${ }^{10}$ de modo que se consideraban indispensables tanto la separación de los niños para evitar el peligro de contagio como la intervención profesional médica.

En sus informes Calamatta señalaba que el tracoma

[...] por un lado ataca al individuo en el órgano esencial para su vida de relación... anulando la actividad productora del enfermo, por otra parte se propaga con terrible genio destructor; de ojo a ojo, de persona a persona; haciendo el mayor número de víctimas entre las familias pobres, donde encuentra el mejor terreno de difusión a causa de la ignorancia, de la miseria, de las condiciones económicas individuales, del ambiente y del hábito de vida doméstica y social contraria a cualquier precepto de higiene ${ }^{11}$.

En ese sentido, otorgaba un significado social a la enfermedad, vinculada a la "clase pobre" y a sus condiciones de existencia, al desconocimiento de hábitos higiénicos y a la recurrencia a formas curativas populares que podían agudizar el cuadro de la enfermedad.

Aunque en un principio primaba la cuestión de que los enfermos debían ser separados, la necesidad de intervenir en una realidad con ambientes distintos, la ciudad y el campo, hizo que las propuestas difirieran acerca de cómo los niños podían permanecer juntos o separados.

Con el objetivo de limitar e impedir la expansión del tracoma en la ciudad, se recomendaban inspecciones sanitarias periódicas en las escuelas, la instalación de dispensarios antitracomatosos y salas de aislamiento en los hospitales; pero

10 Estos preceptos se enunciaron en el informe El tracoma en las Escuelas (1915, p. 9).

11 Como causas que favorecían la difusión del tracoma se enunciaban la aglomeración excesiva de personas en habitaciones reducidas; la escasez de aire, luz y agua; la falta de higiene; la mala alimentación; la ignorancia y las supersticiones populares. Las recomendaciones de la "higiene de la casa y de la persona" eran permanentes y apuntaban a crear una conciencia higiénica, sobre todo en la población pobre, indispensable para el éxito de las acciones (EL TRACOMA..., 1915, p. 9). 
como uno de los centros de difusión de la enfermedad eran los establecimientos escolares, se sostuvo que el medio de profilaxis más eficaz era crear una escuela especial de tracomatosos, donde los niños podían a la vez recibir instrucción y ser curados ${ }^{12}$. La idea, que se concretaría años más tarde, suponía un modelo de separación de los niños enfermos como forma de preservar a los sanos y lograr la declinación de la enfermedad.

En las áreas de la campaña, con menos recursos y más dificultades - vinculadas a la extensión geográfica, la población diseminada y a ciertas resistencias de los habitantes -, las medidas de profilaxis general deberían estar a cargo de "personas aptas", denominación que incluía a médicos, maestros y sacerdotes ${ }^{13}$. Como no era viable un plan de escuelas y dispensarios especiales en el campo y ante la disyuntiva de la expulsión de los tracomatosos de las escuelas - con el costo de la "ignorancia y el incitamiento a la vagancia y al vicio" -; Calamatta se pronunció por el aislamiento en el aula y en los recreos. Se optaba entonces por una salida intermedia, donde los niños enfermos identificados luego de la inspección sanitaria podrían permanecer dentro del ámbito escolar, pero separados de los sanos y reforzando las precauciones con lavajes sistemáticos de bancos y paredes. Y, aunque no puede comprobarse si efectivamente se cumplió, el consejo a las autoridades de las escuelas inspeccionadas era que el aislamiento en las aulas se hiciera sentando a los alumnos enfermos en bancos apartados con la leyenda: "bancos para tracomatosos" (EL TRACOMA..., 1915, p. 27 30). Estas cuestiones inducen a pensar en el cuestionamiento de los límites de la segregación de los enfermos y del estigma que traían el señalamiento y la separación, con interrogantes acerca de los efectos que pudieron llegar a tener en el aprendizaje y en algunas conductas de los niños.

Los informes fueron elevados al Poder Ejecutivo junto con un plan de acción del que participaron el oculista Julio González Lelong y el inspector médico escolar, Manuel de la Fuente, cuyas inspecciones en la Capital dieron un $25 \%$ de granulosos en las escuelas de los suburbios. Este porcentaje los llevaba a denominar a Tucumán como el "paraíso del tracoma", y resaltaban

12 El ejemplo provenía de Italia, donde el mismo Calamatta había atendido una de esas escuelas especiales en Milán entre 1907 y 1909 con resultados satisfactorios y en base a esa experiencia enunció los requisitos que debía tener el establecimiento en cuanto a su ubicación, tipo de personal, necesidad de un dispensario y un especialista a cargo (EL TRACOMA..., 1915, p. 26-27).

13 Los religiosos debían colaborar para combatir supersticiones o concurrencia de los pobladores a curanderos; los maestros dar conferencias, colocar carteles explicativos sobre el tema e intervenir en las curaciones. Informe de los médicos escolares al Sr. presidente del Consejo de Educación (EL TRACOMA..., 1915, p. 29-31). La convocatoria a otros actores - curas, maestros, vecinos, personal de policía y de ingenios azucareros - para cuestiones sanitarias era corriente en las zonas rurales, por la escasez de profesionales médicos. 
como causas principales el clima y las condiciones de hacinamiento y falta de higiene de numerosas familias pobres ${ }^{14}$.

Las intervenciones se encaminaron desde entonces y combinaron la estructura ya existente de Defensa antipalúdica con los médicos inspectores de escuelas y el Consejo de Educación, bajo la supervisión del Consejo de Higiene $^{15}$. De acuerdo a la división de la ciudad en dos secciones, la atención de los niños de las escuelas afectados por la dolencia se haría en un consultorio en el Hospital de Niños, bajo control del facultativo Alejandro Pérez y en el consultorio oftalmológico del Hospital Zenón Santillán, a cargo de González Lelong (EL TRACOMA..., 1915, p. 6-7) ${ }^{16}$.

En esta línea, se realizó un censo escolar de tracomatosos en 1919, que según datos aportados por los médicos citados, constató cerca de 400 casos entre declarados y "dudosos"17. Al mismo tiempo, como una forma de alertar a las autoridades, González Lelong elevó una nota al organismo sanitario municipal, la Asistencia Pública, que fue publicada en la prensa local e incluía nuevas cifras y consideraciones. Como un dato "alarmante", señalaba que de un total de 937 enfermos examinados en el consultorio oftalmológico del Hospital Santillán entre enero y junio, 428 eran tracomatosos (un $45 \%)^{18}$. Advertía que al consultorio

Concurren enfermos de la campaña y pueblos distantes en general gente pobre, y muchas veces llegan a la ciudad sin recursos... Después de pasar la noche en algún umbral o banco de una plaza se presentan a la consulta, donde constatamos que se trata de tracomatosos... Si se admite un

14 Aunque por esto se asimilaba a Tucumán a la región de Catania (Italia), donde el tracoma era de carácter endémico y constituía uno de los principales focos de Europa, los porcentajes variaban según la ubicación de la escuela: si en una de los suburbios llegaba al $25 \%$, en una del centro de la ciudad era de 1/2 (EL TRACOMA..., 1915, p. 34-35).

15 Las medidas recomendadas en las escuelas - aunque no podemos medir su alcance y efectividad - eran entre otras: eximir de asistencia a niños con tracoma hasta que no haya secreción; sentarlos en bancos aislados; obligarlos a lavarse las manos; citar a padres para explicarles el peligro; proveer de agua y medios de higienización; realizar controles médicos al menos dos veces por año (EL TRACOMA..., 1915, p. 37).

16 Estos consultorios, vinculados al Consejo de Educación - que facilitaría un enfermero y elementos de curación -, debían llevar un libro de estadística de los alumnos atendidos y enviar mensualmente al médico escolar el movimiento de los casos, con los datos de los niños, la escuela de procedencia y el tratamiento empleado (EL TRACOMA..., 1915, p. 42).

17 Se advertía que un porcentaje de "dudosos" podría tratarse de enfermos de conjuntivitis catarral (VALDEZ DEL PINO, 1920, p. 125).

18 Bajo el título de "El desarrollo del tracoma", retomaba las conclusiones del profesor Demaría expuestas en el Congreso Internacional de Medicina en 1910, respecto a la necesidad de aumentar consultorios, crear escuelas especiales o habilitar horas destinadas para curar enfermos (EL ORDEN, 19 ago. 1919). 
enfermo de esta clase se dejan de admitir varios de las otras y es por lo que la igualdad de las circunstancias y aún sintiendo compasión infinita por los tracomatosos, dejan los médicos muchas veces de admitirlos (EL ORDEN, 19 ago. 1919).

Esa realidad, a su juicio, hacía imprescindible la creación de una sala para enfermos de la vista, - el contacto con otros enfermos suponía la "exposición al mal" y el contagio - y otras urgentes medidas de parte del gobierno, pero lo cierto es que entonces ya había avances en Tucumán en la consideración del tracoma, con cifras que reflejaban su incidencia en la población; dos consultorios en la ciudad; medidas profilácticas que se aconsejaban aplicar en las escuelas; e intervenciones conjuntas de las autoridades sanitarias y escolares, que de alguna manera prepararon el terreno para otras iniciativas y para la creación de una institución especial.

Mientras a nivel nacional - a excepción del caso de la capital del país - los logros eran incipientes respecto a la identificación y estudios de la patología en las escuelas, en Tucumán los antecedentes señalados confluyeron en la creación de una Escuela de Tracomatosos en 1920, que constituyó un proyecto más elaborado vinculado al tratamiento del tracoma desde la esfera estatal, en la medida en que se fundó en los datos estadísticos recogidos, los informes realizados y el tratamiento sugerido por especialistas. Asimismo, requirió de la decisión política de otorgar un aporte económico, un local y recursos humanos adecuados.

$\mathrm{Al}$ respecto se señalaba que

[...] esta circunstancia, encarece doblemente la trascendencia de esta valiosa acción del actual Consejo de Educación, que viene a combatir uno de los gravísimos males que se han desarrollado en forma visible y alarmante en todo el país, y con caracteres mucho más graves en Tucumán y provincias limítrofes. Esta escuela marcará un nuevo rumbo a seguir e imitar en otras provincias tan castigadas como la nuestra por esta plaga tan peligrosa (VALDEZ DEL PINO, 1920, p. 124).

El autor del proyecto fue el médico Raúl Colombres - también autor de la mencionada ley de enseñanza obligatoria de higiene infantil en las escuelas ${ }^{19}-$,

19 Raúl Colombres (1884-1920) era médico de niños; fue legislador provincial en dos períodos (1914-18 y 1918-22); presidente del Partido Liberal (fundado en 1917) y candidato a gobernador de la provincia al momento de su muerte en 1920. Los homenajes y discursos en sus 
que fundamentó su iniciativa en las graves dimensiones de la enfermedad en la provincia y en la necesidad de combatirla y prevenir su difusión. Al proponer la inclusión de una partida en el presupuesto para la escuela contó con el apoyo del gobernador y de las autoridades escolares, y en consecuencia se logró conseguir un edificio para habilitar las aulas, el consultorio y la sala de curaciones. Así Tucumán se constituía en la primera y única ciudad del país que contaba con una escuela para niños tracomatosos, y de este modo se imponía el concepto de la necesidad de separación de los enfermos.

Acerca del funcionamiento de la institución, dependiente del Consejo General de Educación, la inscripción alcanzada en sus inicios llegó a 165 alumnos, pero para que sus objetivos se cumplieran se buscaron estrategias para facilitar la asistencia de los niños. El Consejo de Educación dispuso proveer pases en los tranvías en aquellos casos que lo justificara la distancia; y para vencer posibles resistencias - adjudicadas a prevenciones por ignorancia o por prejuicio - se hizo llegar a los domicilios "sugestiones verbales y escritas" a fin de conseguir que los padres envíen a sus hijos a la escuela y asistan al consultorio para las curaciones o para obtener medicamentos. Esto respondía no solo al principio de aislar a los niños tracomatosos, sino también a la ocasión de difundir entre las familias los medios para prevenir la infección. Asimismo, además de la atención en el consultorio, a cargo del oculista Julio González Lelong; se asistía con cursos especiales a las maestras, a fin de que adquirieran las herramientas para transmitir a sus alumnos los conocimientos adecuados para el tratamiento que debían seguir (VALDEZ DEL PINO, 1920, p. 123-125).

Según el antecedente de la identificación realizada años antes, los niños que asistían a la escuela provenían de familias pobres ya que los afectados serían de las zonas de los suburbios. En cuanto al local de la escuela, ubicado en un área central de la ciudad, de acuerdo a los testimonios y a las imágenes que reflejan las fuentes, pareció reunir las condiciones adecuadas por sus características edilicias y por las reformas incorporadas, producto de las gestiones realizadas desde la legislatura y de la tarea conjunta de los organismos de salud y de educación de la provincia.

Si bien la Escuela de Tracomatosos "Raúl Colombres"20 contó con financiamiento estatal, la constitución de una Sociedad Protectora aprobada por el

exequias de parte de representantes de la legislatura, del Cuerpo Médico, del Consejo de Educación y de su partido resaltaron sus virtudes como "médico de los grandes y de los humildes" y su obra legislativa como higienista, en particular su iniciativa de la Escuela de Tracomatosos que tendía a "salvar a niños de la muerte", al "mejoramiento de la raza", y a combatir la terrible plaga introducida en el país por la inmigración "sin control" (El ORDEN, 16 en. 1920; 17 en. 1920; 19 en. 1920).

20 Primero denominada "Guillermo Rawson" y luego designada con el nombre de Raúl Colombres en homenaje al autor del proyecto de creación (DIARIO..., 27/4/1922, p. 26-27). 
Consejo de Educación para cooperar con el mantenimiento de la escuela podría reflejar algunas dificultades económicas en sus primeros tiempos y también la continuidad de la contribución de particulares a instituciones vinculadas a lo social en los años $1920^{21}$. En 1923, el establecimiento fue incluido en el presupuesto provincial, destinándose aportes para el pago de las retribuciones ${ }^{22}$, pero resulta llamativo que en ese mismo año, y a casi tres años de su instalación - en 1920 los documentos brindan información y hasta fotos del importante edificio de la escuela y su consultorio -, un informe del Consejo de Educación publicado en la prensa en el que figuran las escuelas de la provincia, incluidas las especiales, no incorpora datos de la escuela de tracomatosos (EL ORDEN, 31 dic. 1923) ${ }^{23}$.

Tampoco el Dr. Gregorio Aráoz Alfaro, en una visita realizada a la provincia como presidente del Departamento Nacional de Higiene, mencionó la institución al referirse a las acciones necesarias contra el tracoma, que requerían una encuesta en todo el país para conocer su extensión y distribución y para la ejecución de un plan profiláctico que en Tucumán contaría con los servicios de Calamata y González Lelong. Seguramente no desconocía la existencia de la escuela pero en su exposición predominaban las preocupaciones por otras patologías, en tanto los mayores esfuerzos e interés estaban en ese momento dirigidos a la lucha antipalúdica y contra la tuberculosis ${ }^{24}$.

Hacia 1927, la partida destinada a la escuela de tracomatosos, que no aparece discriminada en el presupuesto provincial pero formaba parte de los gastos generales de educación, reflejó cierto aumento según indican las Actas

21 Nota del Consejo de Educación a la presidenta de la Comisión protectora sra. Filomena Castro de González Lelong (EL ORDEN, 28 ago. 1922). Nota de agradecimiento por su donación de $300 \$$ al sr Ramón Paz Posse (importe para compra de delantales para los niños) (EL ORDEN, 20 set. 1922).

221 director - $225 \$$-; 1 secretario - $140 \$-; 5$ maestros - $700 \$$ (140\$ cada uno) -; 1 profesor de música $-100 \$-; 1$ enfermera $-90 \$-; 1$ conserje $-60 \$-;$ y para medicamentos $-100 \$$ - , lo que daba un total de 1415\$ (ARGENTINA, 1923).

23 En consonancia con las iniciativas que venimos señalando, desde 1919 existió en Tucumán una Escuela de ciegos, la primera del norte de la República "humanitaria cuanto indispensable institución, que viene a llenar un sentido vacio, en beneficio de los que, la naturaleza, por quien sabe qué designio fatal (nos preguntamos: - ¿tal vez el tracoma?), les ha privado de uno de los órganos acaso más indispensables del hombre: la vista". Bajo protección del gobierno y de una comisión integrada con los presidentes del Consejo de Higiene y del Consejo de Educación, funcionaba en un local reducido, con dos pequeñas aulas y poco personal y material especial didáctico. En 1920 los ciegos de la escuela llegaban a cerca de 70; la enseñanza era con el sistema braile, y aprendían canto, trabajos manuales y labores (EL ORDEN, 6 abr. 1920). En 1922, la prensa destacaba que el apoyo oficial era escaso, se requerían contribuciones particulares y no podían ingresar todos los inscriptos por falta de espacio (EL ORDEN, 28 oct. 1922; 18 nov. 1922).

24 Conversando con el presidente del Departamento Nacional de Higiene (EL ORDEN, 13 oct. 1923). 
del Consejo de Educación ${ }^{25}$. Sin embargo, a mediados de los años 1930 es posible que haya dejado de realizarse el ensayo de separación de niños con tracoma. No tenemos noticias sobre las razones de tal decisión, pero sí nos permite reflexionar sobre que su corta vida implicó que los mismos médicos y maestros se debieron preguntar acerca del éxito de una medida que no impedía a otros niños, en otros colegios, tener tracoma y contagiar a su núcleo familiar, y a la vez, significaba una organización médico sanitaria difícil de obtener con los mecanismos de entonces (traslado de los niños, permanente revisión médica y a la vez, atender a su aprendizaje $)^{26}$.

En suma, el caso de Tucumán permite reflexionar acerca de las vinculaciones entre salud y educación, a través de las preocupaciones por una enfermedad que si no llegaba a dejar ciegos a los niños atacados podía producir la disminución en la visión, con graves consecuencias. No eran ajenas al discurso de las autoridades la gravitación de ideas que apuntaban a la educación y a mejorar la salud para fortalecer a la población para el trabajo productivo; el convencimiento de que la escuela podía ser un foco de extinción y no de infección - esto era "combatir el mal en su fuente de origen" -; y de que los agentes del cambio debían ser los profesionales médicos a cargo de las inspecciones y tratamientos curativos. Más allá del impacto del número de afectados, la visibilidad del tracoma y sus efectos en la salud y la economía, así como la presencia decisiva de especialistas y la incidencia del desarrollo sanitario a nivel local, hicieron que fuera parte de la agenda oficial de modo más temprano y más contundente al de otros centros del país y con anterioridad a que el Departamento Nacional de Higiene expandiera sus esfuerzos en la década de $1930^{27}$.

25 Director 270\$; secretaria 168\$; 5 maestras $168 \$$ cada una; profesor de música $120 \$$, enfermera 100\$; conserje 60\$ (ACTAS..., 14/10/1927, p. 35).

26 En 1924 se resalta el ejemplo y la existencia de la escuela especial en Tucumán (CONFERENCIA..., 1924, p. 178); en 1937 y 1938, los informes señalan la importancia de la profilaxis en el norte argentino y el establecimiento de un dispensario en Tucumán (BOLETÍN..., 1937, p. 518) y la intensificación de medidas en el medio escolar - con datos de escuelas y alumnos examinados -, pero no hacen ninguna referencia a la escuela de tracomatosos en la provincia (BOLETÍN..., 1938, p. 223).

27 Si analizamos la cuestión política, vemos que la creación de la escuela tuvo más que ver con las iniciativas e impulso de los conservadores que del gobernador radical Juan B. Bascary, quien tuvo que enfrentar la oposición en la legislatura de una facción radical disidente y de sectores conservadores, con amplia mayoría. Estos habían formado el Partido Liberal en 1917, al cual pertenecía Raúl Colombres, autor del proyecto (PÁEZ DE LA TORRE, 1987, p. 619). 


\section{Juntos, pero sanos. La extensión del brazo sanitario a las escuelas}

Los gobiernos conservadores argentinos de los años treinta y cuarenta se preocuparon mucho más por extender el brazo estatal para llegar a todos los escolares. En 1939, la Ley Nacional n. ${ }^{\circ} 12.558$ sobre "Protección a los niños en edad escolar" establecía que dos entidades hasta entonces con objetivos disímiles, como lo habían sido la higiene y la educación, y cuyas agencias principales se encontraban en diferentes Ministerios estuviesen coordinadas. Las cuestiones de salud estaban concentradas en el Departamento Nacional de Higiene y luego Secretaría de Salud y bajo el ala del Ministerio de Interior; las de educación, estaban a cargo del Consejo Nacional de Educación, en el Ministerio de Justicia e Instrucción pública. La legislación preveía la constitución de una Comisión con los presidentes de ambas entidades (COMISIÓN..., 1939). De acuerdo a Billorou (2011), la ayuda social y sanitaria hasta este momento estaba concentrada en los niños "anormales" y débiles. La nueva legislación implicaba aplicar mecanismos para cubrir a todos los escolares y considerar sobre todo a los de las Provincias y territorios del Norte argentino a través de un apoyo especial y a partir de esa noción, sentar las bases en lo que luego fue el principal slogan del peronismo: "Los únicos privilegiados son los niños".

¿Cuál fue la implicancia para la atención de los afectados de tracoma? A mediados de los años treinta, la enfermedad asumió una nueva importancia para el principal organismo sanitario nacional y también para algunas de las provincias implicadas. En 1937, el Departamento Nacional de Higiene había organizado una campaña contra el tracoma, detectando en las escuelas un 14\% de niños enfermos (6.983 de 49.626 examinados, en CÁRDENAS, 1942). Y la Ley nacional anteriormente mencionada explicitaba que los maestros tenían determinadas obligaciones respecto a la salud de los escolares y sobre todo, que estaban autorizados a practicar curaciones a niños con tracoma en las aulas. Es curioso que de todas las enfermedades que en ese momento estaban presentes en la agenda sanitaria - como la tuberculosis, la lepra, la malaria, la sífilis, el bocio, entre muchas más -, la única que se menciona específicamente en la legislación es a la conjuntivitis granulosa (ARGENTINA, 1939).

Estas cuestiones merecieron una atención más destacada dentro de la máxima autoridad médica del país a partir de ese momento, así como de una coordinación general para imprimirle el carácter sanitario y nacional que se refleja en dos cuestiones: en primer lugar, en la sanción de la Ley Nacional n. ${ }^{\circ}$ 12.317 sobre denuncia obligatoria de enfermedades contagiosas que incluía a esta patología oftálmica. En segundo lugar, en la organización institucional de la 
Sección Oftalmológica de Profilaxis del Tracoma y oftalmias dentro del DNH, cuyo organigrama rezaba la existencia dentro de esta dependencia, sita en la Capital Federal, de quince dispensarios en Capital Federal y en las provincias de Corrientes, Santiago del Estero, Tucumán y en el Territorio Nacional del Chaco. La Sección dependía de la Secretaría General y estaba al nivel de otras centrales para la política sanitaria de entonces, como las de paludismo, tuberculosis, anquilostomiasis, peste y venéreas, lo cual hace pensar que se intentaba dotar institucionalmente al tracoma de similar especialización burocrática ${ }^{28}$.

En 1938, se llevó adelante la Primera Reunión del personal Técnico de los Servicios de Paludismo, Tracoma y Anquilostomiasis del Norte del País, que reunía el personal técnico de los Territorios Nacionales del Chaco, Formosa y Misiones y las Direcciones correspondientes al Norte y la provincia de Corrientes. Se trataba de la primera reunión de este tipo en el país que implicaba focalizar en las regiones más afectadas por la enfermedad, y los "médicos del tracoma" serían los encargados de difundir las principales medidas de profilaxis y terapéutica.

Las intervenciones estuvieron a cargo de Jefes de Servicio e inspectores del tracoma en el Departamento Nacional de Higiene y de los médicos de zona de las provincias invitadas, y coincidieron en que la enfermedad era una de las más difundidas a nivel mundial - un $15 \%$ de la población de todo el globo, cerca de 100 millones de personas. Dentro del país, de acuerdo a uno de los facultativos, Jiménez, las cifras eran alarmantes; se citaba entre el 15 al 55\% de la población afectada en las provincias y territorios del Norte argentino (PRIMERA..., 1938, p. 62-69).

La idea de los oftalmólogos del Departamento era capacitar a los médicos para que advirtieran los signos iniciales del tracoma y luego fueran capaces de diagnosticar a los pacientes, quienes rara vez interpretaban sus síntomas en los primeros estadios y concurrían a los dispensarios cuando ya era muy tarde y podían perder la vista. Ante la falta de consultas espontáneas, "la tarea era ir en busca del enfermo" y a la vez, llegar a definir los diagnósticos, ya que la conjuntivitis granulosa podía confundirse con otras infecciones bacterianas o virales agudas e igualmente molestas pero no tan graves (PRIMERA..., 1938, p. 62-69).

Detengámonos un momento en las palabras vertidas por José Toja, Jefe de Servicio y médico del tracoma en Tucumán señalaba una gradación de la atención: en primer lugar, la observación de todos los potenciales enfermos en

28 Se destacaban los siguientes funcionarios: en Corrientes, el encargado del Dispensario eran los médicos Roberto Iglesias, Antonio Maráttoli, Carlos Goya, Lucio Cabrera, Aníbal Dacunda y Juan Suriano Soto; en Chaco, Máximo Soto; en Santiago del Estero, Juan Anelli; en Tucumán, José Toja (ORGANIGRAMA, 1938). 
hogares, escuelas e ingenios a cargo de visitadoras de higiene, luego una revisión de los "médicos de zona" de los casos sospechosos. Se debía entrenarlos si no eran oftalmólogos en el uso de la lupa binocular de Berger; la visita del inspector del tracoma resultaría necesaria cuando "[...] el médico práctico no ha hecho la especialidad y hasta tanto haya adquirido experiencia en el diagnóstico y el tratamiento" (PRIMERA..., 1938, p. 101-102).

Con algunas variantes, los distintos ponentes de esta reunión, entre los cuales había tenido un lugar preponderante Demaría, por entonces el máximo representante del país en relación al tracoma, acordaban que se trataba de una enfermedad social, fruto de viviendas en pésimas condiciones y de falta de posibilidades higiénicas en escuelas, hogares, talleres e ingenios azucareros (la principal actividad laboral de los jornaleros en Tucumán). De manera enfática, se refirieron al peligro de un aumento de no-videntes, con el consiguiente riesgo para el país y de que era posible la erradicación con un cuidadoso sistema que ponía en sus manos todo el sistema para organizar la profilaxis y el tratamiento ${ }^{29}$.

La discusión fluctuó entre iniciar desde los dispensarios la labor médica hacia los hogares, a través de visitadoras, o hacerlo en las escuelas, a partir de la conexión entre médicos-maestras: la primera implicaba extender el brazo estatal desde los primeros años de vida e intervenir en cierta manera en los "ranchos" antihigiénicos y la segunda, utilizar a las maestras, cuya eficacia ya había sido demostrada como agentes higiénicos en otros espacios nacionales y desde tiempo atrás (DI LISCIA, 2004).

Tanto en el Chaco como en Jujuy, Santiago del Estero, Tucumán y Corrientes, los médicos recomendaban que, dado que se trataba de una enfermedad masiva, debía ser también general la observación de posibles casos y la experiencia de las mujeres podía aprovecharse con grandes beneficios (PRIMERA..., 1938). No vamos a detenernos sobre la subordinación de género que se deriva de esta valoración del trabajo femenino ya que la historiografía ha abundado en esta demarcación de campos y en sus consecuencias sobre las profesiones de enfermería y docencia, pero sí es preciso remarcarla ya que la detección y luego curación de un número de casos cada vez mayor - en todas las fuentes se habla de miles de enfermos - hacía imposible su atención, salvo que se utilizara también un gran número de agentes ${ }^{30}$.

29 Por ejemplo, Villagrán (PRIMERA..., 1938, p. 92) expresó: “[...] el paraíso del tracoma es un rancho pobre y sucio [...] no es que la gente sea débil y mal nutrida sino que la pobreza los obliga a ser sucios". Para Jiménez, otro de los facultativos, la enfermedad se contagiaba entre "desaseados que viven en promiscuidad" y la solución era mejorar las condiciones sociales desde el hogar (PRIMERA..., 1938, p. 62).

30 También en los dispensarios de la Cruz Roja, organizados por los oftalmólogos Demaría, Olle y Alvarez una década antes en Santiago del Estero, las enfermeras y visitadoras realizaban 
En las campañas, se retrata a maestras administrando los colirios en las escuelas e incluso fuera de allí, a todos los vecinos que requieren la medicación y aún en días no laborables. Y cuando no había maestras, se requería a las visitadoras, "muchachas" aún más eficaces para llevar adelante la curación del tracoma que, con anterioridad a la revolución que produjo el uso de las sulfamidas, implicaba un tratamiento largo, periódico y doloroso (PRIMERA..., 1938 , p. 154) ${ }^{31}$.

Uno de los debates sobre el tracoma era la forma del contagio, cuando se habla en distintos medios periodísticos, a través de campañas focalizadas y en las escuelas de la transmisión de enfermedades sociales, como la sífilis y la tuberculosis. En principio y en relación con la inmigración, se insistió en que la expansión era a raíz del contagio en las largas travesías por mar, donde se carecía casi totalmente de medios para la higiene corporal. Y el examen médico escolar descubría esta enfermedad entre los niños, por lo que se sostuvo la idea de separar a aquéllos con tracoma y continuar el largo tratamiento para extirparlo en escuelas especiales en las provincias donde era considerado como endémico. Este era el concepto que había sostenido el proyecto y los argumentos para una instalación temprana de una Escuela de tracomatosos en Tucumán, como ya analizamos.

Hacia finales de los años treinta, se consideraba que no era necesaria la separación de niños y que podían continuar en las escuelas comunes, ya que la patología podía muy bien controlarse a través de la tarea conjunta de maestras y médicos porque el contagio se producía mucho antes de que los escolares ingresaran a las instituciones, es decir, a través de una convivencia permanente y no ocasional. Para el mayor especialista argentino, la existencia de la enfermedad en el país "no es seguramente una nota de progreso ni de cultura"; era definitivamente una enfermedad social porque atacaba a un gran número de personas, "[...] y debe ser comprendida dentro de las leyes de asistencia social, porque sólo por excepción conduce a la ceguera pero es crónica, larga, grave, disminuye para el trabajo y conduce a la miseria" (DEMARÍA, 1938, p. 565-566).

De esta manera, la enfermedad quedó enlazada dentro de la agenda de los médicos del interior, en una lucha que los hacía indispensables: ellos eran los encargados de prevenir, y si no era posible evitarla, detectar y luego realizar la terapéutica de la enfermedad. El proceso debía iniciarse en distintos lugares, pero

las curaciones, estableciendo una metodología modélica para el tratamiento oftálmico del tracoma incluso fuera del país (LA HISTORIA..., 1942, p. 127-128).

31 Se menciona la aplicación de sulfato de cobre con un lápiz, cauterizaciones con nitrato de plata, pomadas y colirios en las primeras fases, y tratamiento quirúrgico en las últimas (ARGAÑARAZ, 1932, p. 130). 
sobre todo, en las escuelas, y dadas las características del tracoma y su dispersión, aislar a los niños contagiados no resolvía el problema. La solución estaba de la mano de la extensión de la salud en todas las unidades educativas, y sobre todo, en la expansión de conductas higiénicas y preventivas. Tal cuestión definió así la postura inicial de la segregación por una que reconocía las problemáticas sociales acuciantes de familias de sectores populares en el interior argentino. El caso de una escuela para tracomatosos en Tucumán fue por lo tanto excepcional y denota las propuestas sanitario-sociales de un sector político conservador y de algunos profesionales, muy diferentes de las que primaron en las decisiones de las agencias sanitarias y educativas de los años treinta a nivel nacional y que pusieron la base sobre la extensión del brazo médico hacia un conjunto social mayor en los años siguientes.

\section{REFERENCIAS}

ACTAS del Consejo de Educación de la provincia de Tucumán, 1927.

ARGAÑARAZ, R. Manual práctico de oftalmología. Buenos Aires: El Ateneo, 1932.

ARGENTINA. Ley n. ${ }^{\circ}$ 1.345. Presupuesto General de Gastos de la Administración provincial para el año 1923. Tucumán, 1923.

. Ley $n .^{\circ} 12.558$. Protección de niños en edad escolar. Instituciones complementarias de la educación común. Ministerio Justicia e Instrucción Pública de la República Argentina, Comisión Nacional de Ayuda Escolar, 1939.

ARMUS, D. Las colonias de vacaciones. De la higiene a la recreación. In: SCHARAGRODSKY, P. (Ed.). Miradas médicas sobre la cultura fisica en Argentina, 1880-1970. Buenos Aires: Prometeo Libros, 2014. p. 179-181.

BARBIERI, A. El tracoma en la República Argentina. Anales del Departamento Nacional de Higiene, Buenos Aires, v. XXX, 1924.

BELMARTINO, S. La atención médica argentina en el siglo XX. Instituciones y procesos. Buenos Aires: Siglo XXI Editores, 2005.

BILLOROU, M. J. La labor de la Comisión Nacional de Ayuda Escolar (1938-1943): encarar la acción en su verdadero concepto de imperativo social. In: COSSE, I. et al. (Org.). Infancias: políticas y saberes en Argentina y Brasil, siglos XIX y XX. Buenos Aires: Teseo, 2011. p. 207-233.

BOLETín de la Oficina Sanitaria Panamericana, Series: 1937 y 1938. 
BRAVO, M. C.; CAMPI, D. Elite y poder en Tucumán, Argentina, segunda mitad del siglo XIX. Problemas y propuestas. Secuencia, Ciudad de México, n. 47, p. 75-104, 2000.

CAMPI, D. Economía y sociedad en las provincias del norte. In: LOBATO, M. (Dir.). Nueva Historia Argentina. El progreso, la modernización y sus límites (1880-1916). Buenos Aires: Sudamericana, 1999. Tomo V. p. 72-117.

CÁRDENAS, A. La lucha antitracomatosa en Santiago del Estero. Primer Congreso de Medicina de Santiago del Estero, 1942, no paginado.

COMISIÓN Nacional de Ayuda Escolar. Ley 12.558 (Texto de la ley). Buenos Aires: Ministerio de Justicia e Instrucción Pública, 1939. p. 19-22.

CONFERENCIA Sanitaria Panamericana, 1924.

CONI, E. Progrès de l'Hygienie dans la Republique Argentine. Paris: J. B. Baillière, 1887.

DEMARÍA, E. Profilaxis del tracoma en la República Argentina. La Semana Médica, Buenos Aires, año XLV, n. 11, p. 565-73, 1938.

DIARIO de Sesiones, Cámara de Senadores de Tucumán. Sesiones ordinarias y extraordinarias y Asambleas legislativas. Tucumán: Talleres Gráficos La Gaceta, 1922.

DI LISCIA, M. S. Agendas, profesionales y enfermedades: sobre oftalmología y tracoma en Argentina. In: CUENYA, M. A.; ESTRADA, R. (Ed.). Enfermedad, epidemia, higiene $y$ control social. Nuevas miradas desde México y América Latina. Puebla: Dirección de Fomento Editorial, 2013. p. 218-37.

. Desde fuera y desde dentro. Enfermedades, etnias y nación (Argentina, 18801940). In: HOCHMAN, G.; DI LISCIA, M. S.; PALMER, S. (Ed.). Patologías de la patria. Buenos Aires: Lugar Editorial, 2012. p. 124-153.

. Médicos y maestros. Higiene, eugenesia y educación en Argentina, 1880-1940. In: DI LISCIA, M. S.; SALTO, G. (Ed.). Higienismo, educación y discurso en la Argentina, 1870-1940. Santa Rosa: EDULPAM, 2004. p. 37-64.

. Una larga marcha hacia la inclusión. Enfermedades y campañas en el interior argentino (1900-1940). In: LLUCH, A.; MORONI, M. (Ed.). Tierra adentro... Instituciones económicas y sociales en los Territorios Nacionales (1884-1951). Rosario: Prohistoria, 2010. p. 121-139.

EL ORDEN, Tucumán, 1919-1923 [páginas no numeradas].

EL TRACOMA en las Escuelas. Investigaciones y medidas para combatirlo. Tucumán: Tipografía de la Cárcel Penitenciaria, 1915. Biblioteca de la Casa de Gobierno.

FERNÁNDEZ, M. E. Salud y Políticas Públicas en el Tucumán del Entresiglo (18801916). Tesis (Doctorado en Historia) - Facultad de Filosofía y Letras, Universidad Nacional de Tucumán, Tucumán, 2013.

GARCÍA, D. (Ed.). Compilación ordenada de leyes y decretos de la provincia de Tucumán. [s.l.: s.n.], 1934. Tomo I. 
GIOIA, E. La santé au pouvoir. Le trachome au début du XXème siècle et le refoulement des migrants granuleux dans le port de Buenos Aires entre 1908 et 1943. These (Magister) - Institut d Etudes Politiques, Paris, 2013.

GONZÁLEZ LEANDRI, R. Internacionalidad, Higiene y Cuestión Social en Buenos Aires (1850-1910). Tres momentos históricos. Revista de Indias, v. 73, n. 257, 2013. Doi: 10.3989/revindias.2013.002.

. La élite intelectual docente como fracción intelectual seleccionada. Argentina, 1852-1900. Anuario de Estudios Americanos, n. 58, p. 513-534, 2001.

GUTIÉRREZ, J. M.; DIEZ MORI, S. Higiene escolar. El Monitor de la Educación Común, Buenos Aires: Consejo Nacional de Educación, p. 450-472, 1905.

LA HISTORIA del tracoma desde hace cinco mil años. Revista Médica Hondureña, año 12, n. 2, p. 119-128, 1942.

LÁZARO, O. Tres aspectos del gobierno de Lucas Córdoba. Revista de la Junta de Estudios Históricos de Tucumán, Tucumán, p. 25-39, 1968.

LEMA, B.; CALDERÓN, E. Informe del Inspector Nacional de Escuelas en Tucumán, correspondiente al 2. ${ }^{\circ}$ cuatrimestre de 1897. El Monitor de la Educación Común, Buenos Aires: Consejo Nacional de Educación, p. 933-941, 1898.

LIBRO Copiador de Notas del Consejo de Higiene de la provincia de Tucumán, 1897.

LIJÓ PAVÍA, J. Asistencia social de las enfermedades oculares. Revista de Oto-neurooftalmológica y de clínica neurológica sudamericana. Buenos Aires, tomo XII, n. 5, p. 119-129, mayo 1937.

. La inspección oftalmológica en las escuelas de Buenos Aires. El Monitor de la Educación Común, Buenos Aires: Consejo Nacional de Educación, p. 34-41, 1922.

MAGGI ZABALIA, A. El tracoma en la Provincia de Córdoba. Archivos de Oftalmología de Buenos Aires, tomo II, n. 4, p. 239-280, 1927.

. El tracoma en la Provincia de Santa Fé. Boletín de la Oficina Panamericana, v. 15, n. 12 , p. 1168-1169, 1936.

MARKEL, H. The Eyes Haves It: Trachoma, the Percepcion of Disease, The United State Health Service and The American Jewist Inmigration Experience, 1897-1924. Bulletin of the History of Medicine, Baltimore, n. 74, p. 525-560, 2000.

MARTÍNEZ, B.; BUSTAMENTE, J. M. M. Informe de Benjamín Martínez sobre el estudio de la visión en los niños - Higiene escolar. El Monitor de la Educación Común, Buenos Aires: Consejo Nacional de Educación, p. 156-159, 1892.

ORGANIGRAMA. Boletín Sanitario del Departamento Nacional de Higiene. Buenos Aires: Departamento Nacional de Higiene, 1938.

PÁEZ DE LA TORRE, C. Historia de Tucumán. Buenos Aires: Ed. Plus Ultra, 1987. 
PRIMERA Reunión del Personal Técnico de los Servicios de Paludismo, Tracoma y Anquilostomiasis del Norte del País. Boletín Sanitario del Departamento Nacional de Higiene. Buenos Aires: Departamento Nacional de Higiene, 1938.

REGLAMENTO del Cuerpo Médico Escolar. El Monitor de la Educación Común, Buenos Aires: Consejo Nacional de Educación, p. 436-449, 1905.

REGISTRO Oficial de la provincia de Tucumán. Reglamento General para las Escuelas Primarias. Tucumán: [s.n.], 1901.

RODRÍGUEZ DE ANCA. Apuntes para el análisis de las relaciones entre discurso médico y educación. In: DI LISCIA, M. S.; SALTO, G. (Ed.). Higienismo, educación y discurso en la Argentina, 1870-1940. Santa Rosa: Edulpam, 2004. p. 15-35.

ROUGES, A. Plan para combatir el analfabetismo en la provincia de Tucumán. El Monitor de la Educación Común, Buenos Aires: Consejo Nacional de Educación, p. 197-200, 1931.

VALDEZ DEL PINO, A. El Album del Magisterio de la provincia de Tucumán. Tucumán: Consejo de Educación, 1920.

Texto recebido em 15 de outubro de 2014 . Texto aprovado em 21 de outubro de 2014. 
